ULB-PMIF-92/10

\title{
Bianchi Cosmological Models and Gauge Symmetries
}

\author{
Olivier Coussaert ${ }^{1}$ and Marc Henneaux ${ }^{2}$ \\ Faculté des Sciences, Université Libre de Bruxelles, \\ Campus Plaine C.P. 231, B-1050 Bruxelles, Belgium
}

${ }^{1}$ Chercheur IRSIA

2 Maitre de Recherches au Fonds National de la Recherche Scientifique. Also at Centros de Estudios Científicos de Santiago, Casilla 16443,Santiago 9, Chile. 


\begin{abstract}
We analyze carefully the problem of gauge symmetries for Bianchi models, from both the geometrical and dynamical points of view. Some of the geometrical definitions of gauge symmetries (="homogeneity preserving diffeomorphisms") given in the literature do not incorporate the crucial feature that local gauge transformations should be independent at each point of the manifold of the independent variables ( $=$ time for Bianchi models), i.e, should be arbitrarily localizable ( in time). We give a geometrical definition of homogeneity preserving diffeomorphisms that does not possess this shortcoming. The proposed definition has the futher advantage of coinciding with the dynamical definition based on the invariance of the action ( in Lagrangian or Hamiltonian form). We explicitly verify the equivalence of the Lagrangian covariant phase space with the Hamiltonian reduced phase space. Remarks on the use of the Ashtekar variables in Bianchi models are also given.
\end{abstract}

\title{
1 Introduction
}

It is a well known fact that the distinction between global and local symmetries is somewhat subtle in the case of spatially homogeoneous cosmological models of the Bianchi type [1, 2, 3]. There are indeed at least three different ways to define the gauge symmetries 円:

(i) geometrically, as those diffeomorphisms that preserve manifest homogeneity;

(ii) dynamically, from a spacetime covariant point of view, as those transformations that leave the action invariant and that are local in time; and

(iii) dynamically, from the Hamiltonian point of view, as those canonical transformations that are generated by the first class constraints.

\footnotetext{
${ }^{1}$ We restrict the analysis of this paper to class A Bianchi cosmologies as these are the only ones for which one can inject the homogeneous form of the metric in the action prior to computing the variationnal derivatives [4, 5, 6]
} 
It is not a priori clear that these three different points of view should yield identical conclusions. Futhermore, while the dynamical approaches based on (ii) and (iii) yield unambiguous answers, the geometric approach is not so well defined since it depends on what one means exactly by "diffeomorphisms preserving manifest homogneity".

The purpose of this paper is to show that the three different concepts of gauge symmetries are actually the same provided one adopts a natural definition of "homogeneity preserving diffeomorphisms". This definition agrees with the one given in [1, 2] but differs from that proposed in [3]. We first consider the metric formulation of Bianchi models and then briefly comment on the formulation in terms of Ashtekar variables.

\section{Geometric definition of gauge symmetries}

\section{$2.1 \quad$ First definition}

Bianchi cosmological models are spacetimes invariant under a three- dimensional group of isometries $\mathrm{G}$ acting simply transitively on three - dimensional spacelike hypersurfaces [7].

In Bianchi models, one is thus given an action of $G$ on the manifold $\mathcal{M}$,

$$
\begin{aligned}
G & \longrightarrow \operatorname{Diff}(\mathcal{M}) \\
R \in G & \longmapsto h_{R} \in \operatorname{Diff}(\mathcal{M})
\end{aligned}
$$

and the spacetime metric $g$ fulfills

$$
h_{R}^{*} g=g
$$

Since the action of $\mathrm{G}$ is part of the given geometric data, it is natural to define the subgroup $H \subset \operatorname{Diff}(\mathcal{M})$ of "homogeneity preserving diffeomorphisms" as the set of diffeomorphisms which preserve the action of $G$, i.e. which commute with each $h_{R}, R \in G$

$$
f \in H \leftrightarrow f h_{R}=h_{R} f \quad \forall R \in G
$$

If one performs a diffeomorphism $k$ that does not belong to $H$, one can detect it by just comparing the action of $G$ before and after $k$ has been performed. The group $H$ defined by (3) is the centralizer of $G$ in $\operatorname{Diff}(\mathcal{M})$. 
In order to determine the general solution of (3), we observe that the manifold $\mathcal{M}$ is foliated by the three-dimensional hypersurfaces $\Sigma$ of transitivity of $G$. It is convenient to introduce a non-vanishing vector field $v$ transverse to these hypersurfaces and such that the one - parameter group $\left\{e x p x^{0} v\right\}$ commutes with $G$,

$$
\left(\exp x^{0} v\right) h_{R}=h_{R}\left(\exp x^{0} v\right)
$$

This can be done by picking an arbitrary curve $\gamma: x^{0} \mapsto \gamma\left(x^{0}\right)$ everywhere transverse to the hypersufaces $\Sigma$. One then constructs the family of curves $x^{0} \mapsto h_{R}\left(\gamma\left(x_{0}\right)\right)$. Since $G$ acts simply transitively, there is one and only one such curve through each point. One defines $v$ to the tangent vector field to the family. The vector field $v$ fulfills (舟) and $x^{0}$ is constant along the hypersurfaces of transitivity.

Let us now choose a basis in the Lie algebra $\mathcal{G}$ of $G$ and take the corresponding Killing vectors $\xi_{a}(a=1,2,3)$ tangent to the hypersurfaces $\Sigma$. The vector fields $v$ and $\xi_{a}$ provide a basis of the tangent space at each point of the spacetime manifold $\mathcal{M}$. One has

$$
\begin{gathered}
{\left[v, \xi_{a}\right]=0} \\
{\left[\xi_{a}, \xi_{b}\right]=C_{a b}^{c} \xi_{c}}
\end{gathered}
$$

where $C_{a b}^{c}$ are the structure constants of $\mathcal{G}$.

Let $X_{a}$ be a basis of invariant vector fields fulfilling also $\left[v, X_{a}\right]=0$,

$$
\begin{gathered}
{\left[v, X_{a}\right]=0} \\
{\left[\xi_{a}, X_{b}\right]=0}
\end{gathered}
$$

One may take $X_{a}=\xi_{a}$ on the curve given above. In that case, on finds in addition to (5)-(6)

$$
\left[X_{a}, X_{b}\right]=-C_{a b}^{c} X_{c}
$$

The most general "infinitesimal" I diffeomorphism commuting with $G$ is determined by a vector field $\xi$ fulfilling

$$
\left[\xi, \xi_{a}\right]=0
$$

\footnotetext{
${ }^{2}$ These are the only ones considered here.
} 
If one expands $\xi$ in the basis $\left\{v, X_{a}\right\}$,

$$
\xi=\mu v+\mu^{a} X_{a}
$$

one gets

$$
\partial_{\xi^{a}} \mu=0, \quad \partial_{\xi^{a}} \mu^{b}=0,
$$

i.e.,

$$
\xi=\mu\left(x^{0}\right) v+\mu^{a}\left(x^{0}\right) X_{a}
$$

Thus, a homogeneity preserving diffeomorphism depends on four functions of $x^{0}$, irrespectively of the homogeneity group and of the spatial topology. This is quite a satisfactory result, since in the non-homogeneous case, a general diffeomorphism depends also on four functions ( which may be nonhomogeneous). Futhermore, the fact that (11) involves arbitrary functions of $x^{0}$ implies that one can arbitrarily localize the homogeneity preserving diffeomorphisms in time ( there is, of course, no hope to localize them in space in a spatially homogeneous context). This localizability property appears to be an essential feature of gauge (="local") transformations.

We close this subsection by observing that (11) acts though the adjoint action on the invariant frame $\left\{v, X_{a}\right\}$,

$$
\begin{array}{r}
\delta v=[\xi, v]=-\frac{d \mu}{d x^{0}} v-\frac{d \mu^{a}}{d x^{0}} X_{a} \\
\delta X_{a}=\left[\xi, X_{a}\right]=-\mu^{b} C_{a b}^{c} X_{c}
\end{array}
$$

This yields

$$
\begin{array}{r}
\delta g_{00}=\mu \frac{d g_{00}}{d x^{0}}+2 \frac{d g_{00}}{d x^{0}}+2 g_{0 a} \frac{d \mu^{a}}{d x^{0}} \\
\delta g_{0 a}=\mu \frac{d g_{0 a}}{d x^{0}}+g_{0 a} \frac{d \mu}{d x^{0}}+g_{a b} \frac{d \mu^{b}}{d x^{0}}+\mu^{d} C_{d a}^{b} g_{0 b} \\
\delta g_{a b}=\mu \frac{d g_{a b}}{d x^{0}}+\mu^{d}\left(C_{d a}^{c} g_{c} b+C_{d b}^{c} g_{a c}\right)
\end{array}
$$

for the components $g_{\lambda \mu}\left(x^{0}\right)$ of the invariant metric in the invariant frame $\left\{v, x_{a}\right\}$

The transformations (13) are irreductible if and only if the adjoint representation is faithful, i.e., if the center of $\mathcal{G}$ vanishes. Otherwise, there exist non trivial gauge parameters for which the transformations (13) are zero. 
These are given by $\mu=0, \frac{d \mu^{a}}{d x^{0}}=0$ with $\mu^{a}$ a non zero solution of $\mu^{a} C_{a b}^{c}=0$. An irreductible description of the gauge transformations can be obtained as follows. Let $\xi_{A}$ be a basis of the center of $\mathcal{G}\left(C_{A b}^{d}=0\right)$ and $\xi_{\alpha}$ supplementary vectors so that $\left\{\xi_{A}, \xi_{\alpha}\right\}$ is a basis of $\mathcal{G}$. On can replace $\frac{d \mu^{a}}{d x^{0}}$ by the gauge parameter $\epsilon^{A}$ without violating locality in time ( since $\mu^{A}$ does not appear in $(13)$ ), which yields

$$
\begin{array}{r}
\delta g_{00}=\mu \frac{d g_{00}}{d x^{0}}+2 \frac{d g_{00}}{d x^{0}}+2 g_{0 A} \epsilon^{A}+2 g_{0 \alpha} \frac{d \mu^{\alpha}}{d x^{0}} \\
\delta g_{0 a}=\mu \frac{d g_{0 a}}{d x^{0}}+g_{0 a} \frac{d \mu}{d x^{0}}+g_{a B} \epsilon^{B}+g_{a \beta} \frac{d \mu^{\beta}}{d x^{0}}+\mu^{\delta} C_{\delta a}^{b} g_{0 b} \\
\delta g_{a b}=\mu \frac{d g_{a b}}{d x^{0}}+\mu^{\delta}\left(C_{\delta a}^{c} g_{c b}+C_{\delta b}^{c} g_{a c}\right)
\end{array}
$$

(For more information on reductible gauge transformations, see for instance [8] chapter III).

\section{$2.2 \quad$ Second definition}

A weaker form of "homogeneity preserving diffeomorphisms" has been proposed in [3]. These are the diffeomorphisms which map any spatially homogeneous metric on a spatially homogeneous metric. For this to be the case, one must have in place of (8)

$$
\left[\xi, \xi_{a}\right]=\mu_{a}^{b} \xi_{c}
$$

where the matrix $\mu_{a}^{b}$ is constant. Clearly, any solutions of (8) is also a solution of (15). The converse is not true for non compact cosmologies of type $\underline{\underline{I}}, \overline{I I}$, $\overline{V I}_{0}$ or $\underline{V I I}_{0}$ [3]. In finite term, the equation (15) becomes

$$
f h_{R} f^{-1}=h_{R^{\prime}}
$$

Thus, $f$ is a homogeneity preserving diffeomorphism according to the second defintion if and only if its belongs to the normalizer of $G$ in $\operatorname{Diff}(\mathcal{M})$.

Let us now determine which solutions of (15) can be localized in an arbitrarily small spacetime interval. If $\xi\left(x^{0}\right)$ is zero for $x^{0} \notin\left[x_{1}^{0}, x_{2}^{0}\right]$, one has $\mu_{a}^{b}=0$ since $\left[\xi, \xi_{a}\right]=0$ for $x^{0} \notin\left[x_{1}^{0}, x_{2}^{0}\right]$ and $\mu_{a}^{b}$ is constant. Hence, the only homogeneity preserving diffeomorphisms of [3] that can differ from the identity in a finite time interval are precisely the homogeneity preserving 
diffeormorphisms of the previous subsection. If one feels that the concept of "gauge transformation" ( "of the second kind " as one used to say in the old days) should involve in a crucial manner the freedom to change the local reference frame independently at each time ( localizability of the transformation), then, one must impose the condition $\mu_{b}^{a}=0$ in (15) and one falls back on the first definition given previously. This point has not be addressed in [3].

\section{$3 \quad$ Lagrangian definition of gauge symmetries}

\subsection{Gauge transformations}

The previous considerations are purely kinematical and do no refer to the action principle. To decide the issue of which geometrical definition is preferable, one should introduce physical considerations, namely, study the transformation properties of the action principle.

The equations for the Bianchi models of class A can be derived from the Hilbert action in which the symmetry of the metric is enforced before one computes the variationnal derivatives ("reduced variationnal principle") [5, 6]

$$
S\left[g_{a b}, N\left(x^{0}\right), N^{a}\left(x^{0}\right)\right]=\int d x^{0} L\left(g_{a b}, \frac{d g_{a b}}{d x^{0}}, N, N^{a}\right)
$$

The lagrangian contains reference to the homogeneity group through the structure constants $C_{b c}^{a}$ that appear as parameters. It explicit form is [5, [6]

$$
L\left(g_{a b}, \frac{d g_{a b}}{d x^{0}}, N, N^{a}\right)=\int d x^{3} N \sqrt{g}\left(R-K_{c}^{c} K_{d}^{d}+K_{c}^{d} K_{d}^{c}\right)
$$

where:

$$
\begin{array}{r}
R=-\frac{1}{2}\left(2 C^{c}{ }_{c d} C^{a}{ }_{a}{ }^{d}+C^{d c a} C_{c d a}+\frac{1}{2} C^{c d a} C_{c d a}\right. \\
K_{a b}=\frac{1}{2 N}\left[\left(C_{a b}{ }^{c}+C_{b a}{ }^{c}\right) N_{c}-\frac{d g_{a b}}{d x^{0}}\right]
\end{array}
$$

with:

$$
\begin{gathered}
C_{a b c}=g_{a d} C_{b c}^{d}, C^{a}{ }_{a}^{c}=g^{c d} C^{a}{ }_{a d} \\
C^{a b c}=g^{b d} g^{c e} C^{a}{ }_{d e}
\end{gathered}
$$


Note that the shift $N^{a}$ appears only through the combinaision $N^{a} C_{a b}^{c}$, so that the components of $N^{a}$ in the center drop out.

A gauge transformation of (16) is a transformation of the dynamical variables which must fulfills two conditions [8]:

(i) it leaves the action invariant ( up to a boundary term); and

(ii) it is localizable; i.e., it must depend on arbitrary functions ofthe coordinates, which can be chosen so that the transformations reduce to the identity outside a finite region. In the present case where we impose spatial homogeneity, the independent coordinates reduce to $x^{0}$ and a finite region is typically a finite interval $\left[x_{1}^{0}, x_{2}^{0}\right]$.

It is the second point that makes the distinction between gauge ("local") transformation and global symmetry transformations. Gauge symmetries lead to non uniqueness of the Cauchy problem while global symmetries do not.

It should be stressed that the definition (i)-(ii) of the gauge symmetries isentirely "covariant" i.e., formulated from a spacetime point of view. The fact that $x^{0}$ plays a priviledged role is a result of the spatial homogeneity alone and not not of the Hamiltonian splitting of spacetime into space and time.

It is easy to verify that the transformations (13) or (14) when rewritten in terms of $g_{a b}$, the lapse and the shift, leave the action (16) invariant. Furthermore, as we have discussed, they are local in the sense of (ii). Thus, they define gauge symmetries. They actually provide a complete set of gauge symmetries, because once the gauge freedom (13) is frozen- say by imposingg $N=1, N^{a}=0$ - the equations of motion following from (16) have a unique solution. There is no futher degeneracy besides the one implied by (11). [For more on the general description of gauge symmetries, see [8] ]. Accordingly, there is complete agreement between the first geometric definition of gauge symmetries and the dynamical definition based on the Lagrangian.

The extra symmetries which the second geometrical definition allows (elements of the normalizer of $G$ which are not in the centralizer) fail to be dynamical gauge symmetries because, as we have already emphasized, they do not fulfill (ii). Hence, they lead neither to a degeneracy of the Lagrangian nor to an indeterminacy of the Cauchy problem. Trying to "gauge fix" them 
is unnecessary to get an unambiguous evolution and would remove true degrees of freedom. Even though these symmetries are not gauge symmetries, they do define, however, global symmetries as we now discuss.

\subsection{Global symmetries}

The transformations (15) with $\mu_{a}^{b} \neq 0$ induce the following changes of the dynamical variables ,

$$
\begin{array}{r}
\delta N=0 \\
\delta N^{a}=a_{b}^{a} N^{b} \\
\delta g_{a b}=-a_{b}^{c} g_{c b}-a_{b}^{c} g_{a c}
\end{array}
$$

where $a_{b}^{a}$ is a constant matrix belonging to the Lie algebra of the automorphism group of $\mathcal{G}$. The transformations (18) leave the action (16) invariant provided $\operatorname{Tr} a=0$ and hence, define global symmetries when $\operatorname{Tr} a=0$. By Noether theorem, they give non vanishing conserved charges.

A crucial feature of the transformations (18) of the dynamical variables is that they are well defined even if the spacial sections are compact. In that case, they cannot be induced by a globally defined diffeomorphism[3], but still, they make perfect sense. Futhermore, they take exactly the same form irrespectively of the topology. From the dynamical point of view, they should always be regarded as global symmetries since they cannot be localized in $x^{0}$ by a choice of the parameters $a_{b}^{a}$. In the type I case, the corresponding conserved charges account for the integrability of the model [1, 2].

\subsection{Counting degrees of freedom- Covariant phase space}

The number of degrees of freedom of a gauge theory can be counted directly from the Lagrangian, without having to go through the Hamiltonian analysis. It is equal to (half) the dimension of the covariant phase space, defined to be the space of solutions of the equations of motion modulo the gauge transformations [9, 10, 11, 12].

Now, a solution of the equation of motion is completely characterized by: (i) a choice of lapse $N\left(x^{0}\right)$ and shift $N^{a}\left(x^{0}\right)$; and (ii) initial data $g_{a b}\left(x^{0}=0\right)$, 
$\frac{d g_{a b}}{d x^{0}}\left(x^{0}=0\right)$ at $x^{0}=0$ (say) fulfilling the constraint equation

$$
\begin{array}{r}
G^{00}=\frac{\sqrt{\left(N^{2}-N^{s} N_{s}\right)}}{N^{2}}\left(R+K_{i}^{i} K_{j}^{j}-K_{j}^{i} K_{i}^{j}\right) \\
G^{0 i}=\frac{2}{N}\left(-C^{l}{ }_{l}^{i} K_{j}^{j}+C_{l j}^{i} K^{l j}\right)+\frac{N^{i}}{N^{2}}\left(R+K_{i}^{i} K_{j}^{j}-K_{i j} K^{i j}\right)
\end{array}
$$

The number of independent equations (19) is equal to $4-\operatorname{dim}($ center $\mathcal{G})$. By a choice of gauge, on can take $N=1$ and $N^{a}=0$. The residual gauge transformations acting on the initial data $g_{a b}, \frac{d g_{a b}}{d x^{0}}$ take the form

$$
\begin{array}{r}
\delta g_{a b}=\mu \frac{d g_{a b}}{d x^{0}}+\mu^{\delta}\left(C_{\delta a} g_{c b}+C_{\delta b}^{c} g_{a c}\right) \\
\delta \frac{d g_{a b}}{d x^{0}}=\mu\left[-2 R_{a b}-\frac{1}{2} \frac{d g_{c d}}{d x^{0}} g^{c d} \frac{d g_{a b}}{d x^{0}}+\frac{d g_{b l}}{d x^{0}} g^{k l} \frac{d g_{a k}}{d x^{0}}\right]+\mu^{\delta}\left(C_{\delta a}^{c} \frac{d g_{c b}}{d x^{0}}+C_{\delta b}^{c} \frac{d g_{a} c}{d x^{0}}\right)
\end{array}
$$

and involve effectively $4-\operatorname{dim}($ center $\mathcal{G})$ parameters ( recall that $\mu^{a}=\left(\mu^{A}, \mu^{\delta}\right)$ where $\mu^{A}$ is in the center). Hence, the dimension of the space of solutions of the equations of motion modulo the gauge-transformation is equal to 12 (number of $g_{a b}$ and $\frac{d g_{a b}}{d x^{0}}, N$ and $N^{a}$ do not count since they can be "gauged away") minus 4 - $\operatorname{dim}($ center $\mathcal{G}$ ) ( number of independent transformations (20). The number of degreees of freedom is half this count,

$$
\begin{aligned}
n & =\frac{1}{2}\{12-2[4-\operatorname{dim}(\text { center } \mathcal{G})]\} \\
& =2+\operatorname{dim}(\text { center } \mathcal{G})
\end{aligned}
$$

The count (21) agrees with the count of [8], Chapter 3, where it is shown that $n$ is equal to the number of dynamical variables ( here $10=$ number of $\left.g_{\mu \nu}\right)$ minus the number of times the gauge transformations strike. A gauge transformation strikes $\mathrm{n}$ times if the corresponding gauge parameter together with its time derivatives up to order n-1 appear effectively in the gauge transformations]. Here, $\mu$ and $\mu^{\alpha}$ strike twice, but the gauge parameters $\mu^{A}$ in the center strike only once. Hence, again,

$$
\begin{aligned}
n & =10-2\{1+3-[\operatorname{dim}(\text { center } \mathcal{G})]\}-\operatorname{dim}(\text { center } \mathcal{G}) \\
& =2+\operatorname{dim}(\text { center } \mathcal{G})
\end{aligned}
$$


The symplectic structure on the covariant phase space is determined by the boundary term in the variation in the action. One finds here (on-shell)

$$
\delta S=\left[\Pi^{a b} \delta g_{a b}\right]_{x_{1}^{0}}^{x_{0}^{0}}
$$

with

$$
\Pi^{a b}=\sqrt{g}\left(g^{a b} K_{i}^{i}-K^{a b}\right)
$$

Thus, the symplectic two-form on the covariant phase space is the two- form induced by the canonical two-form

$$
\delta \Pi^{a b} \wedge \delta g_{a b}
$$

on the quotient of the surface (19) by the transformations (20). [ One easily verifies that the induced two-form on the surface (19) has a rank equal to $4-\operatorname{dim}($ center $\mathcal{G})$, with zero eigenvectors determined by (20). It is thus regular on the quotient space.]

\section{$4 \quad$ Hamiltonian analysis}

The reader familiar with the hamiltonian analysis of Bianchi models will certainly have noticed that the above results, obtained from a spacetime covariant point of view, are just the same as the Hamiltonian results (see e.g. [2, 3]). The $\Pi^{a b}$ are the momenta conjugate to $g_{a b}$, the constraints (19) are the Hamiltonian constraints and are first class. The transformations (201) are just the transformations which they generate. The details may be found in the literature and will not repeated here. We can thus conclude not only that the various concepts of gauge transformations do coincide, but also, that the covariant phase space is identical (as symplectic manifold) with the Hamiltonian reduced phase space. This later point is of course not an accident since it is been established on general grounds in [13].

\section{Coset spaces}

One can generalize Bianchi models by assuming local homogeneity instead of global homogeneity. In the globally homogeneous case, the surface of transitivity can be identified with the homogeneity group $G$. In the locally 
homogeneous case, one replaces the surfaces of transitivity $G$ by the quotient space $G / K$ where $K$ is a discrete subgroup of $G$ acting on $G$ properly discontinously. The diffeomorphisms of $G$ that do not commute with $K$ fail to globally defined on the quotient space. Homogeneous objects do remain welldefined, however. To study coset spaces, it is convenient to lift everything from $G / K$ to $G$. One then applies the techniques of the previous sections. One finally takes the quotient at the very end. Since the gauge transformations are generated by the vector fields $X_{a}$, which commute with $\xi_{a}$ and hence with $G$ and $K \subset G$, they are well defined in the quotient.

\section{Remarks on Bianchi models and Ashtekar variables}

Recently, Ashtekar has introduced new variables in terms of which the constraints of general relativity take a polynomial form ( see e. g. the reviews $[14,15])$. In order to gain insight into those variables, various authors have reformulated Bianchi models in terms of them [16, 17]. An interesting question then arises: what is the most general form of the Ashtekar variables compatible with homogeneity? We first address this question.

A SO(3)-connection $A_{(a) k}$ and a triad $e_{(a) k}$ are homogeneous if and only if the images $h_{R}^{*} A_{(a) k}$ and $h_{R}^{*} e_{(a) k}$ of $A_{(a) k}$ and $e_{(a) k}$ differ from $A_{(a) k}$ and $e_{(a) k}$ by a local $\mathrm{SO}(3)$ - transformation [18, 19, 20, 21, 22

$$
\begin{array}{r}
h_{R}^{*} A_{k}=\psi A_{k} \psi_{R}^{-1}+\psi_{R} \partial_{k} \psi_{R}^{-1} \\
h_{R}^{*} e_{k}=\psi_{R} e_{k}
\end{array}
$$

where $\psi_{R}$ is a spacerime-dependent element of $\mathrm{SO}(3)$ that depend also on $R \in G$. If we assume that the triads are non-degenerate, or degenerate at most at isolated points, one infers from (27), (28) and $h_{R_{1} R_{2}}^{*}=h_{R_{1}}^{*} h_{R_{2}}^{*}$ that

$$
\psi_{R_{2} R_{1}}(P)=\psi_{R_{2}}\left(h_{R_{1}} P\right) \psi_{R_{1}}(P)
$$

so that the difficulties pointed out in [23] and characteristic of Abelian gauge groups do not occur. One can then apply the general techniques developed in [24, 25]. Since the group $G$ acts simply transitively, one may define $\chi(P)$ as

$$
\chi(P)=\psi_{R}^{-1}\left(P_{0}\right)
$$


where $P_{0}$ is a fiducial reference point on the surfaces of transitivity to which $P$ belongs ( for instance, one can take it to be the intersection point of $\Sigma$ with the curve $\gamma$ ) and $R$ is the unique element of $G$ such that $h_{R}\left(P_{0}\right)=P$.

Let us define $\tilde{A}$ and $\tilde{e}$ by making the $\mathrm{S} 0(3)$ gauge transformation generated by $\chi$,

$$
\begin{array}{r}
\tilde{A}=\chi A \chi^{-1}+\chi d \chi^{-1} \\
\tilde{e}=\chi e
\end{array}
$$

There, one finds from $(27),(28),(29)$ and $(\sqrt{30})$ that $\tilde{A}$ and $\tilde{e}$ are strictly invariant,

$$
\begin{array}{r}
h_{R}^{*} \tilde{A}=\tilde{A} \\
h_{R}^{*} \tilde{e}=\tilde{e}
\end{array}
$$

There is thus no loss of generality in assuming the Ashtekar variables to be strictly homogrneous. This confirms by a geometrical method the findings of [16, 17]. In the invariant frames $\left\{X_{a}\right\}$, the components of $\tilde{A}_{(a) k}$ and $\tilde{e}_{(a) k}$ depend only on $x^{0}$. The Einstein equations define a problem of classical mechanics, as in the metric formulation.

The definition of the gauge symmetries proceeds then as above. These symmetries are found to include besides the homogeneity preserving diffeomorphisms of the metric formulation, the local $\mathrm{SO}(3)$ internal transformations acting on the internal indices. There is again complete agreement between the first geometrical definition and the dynamical definitions.

The analysis can be extended to the discussion of discrete symmetries (see [26, 27]) provided we assume the connection to be an $\mathrm{O}(3)$-connection rather that an $\mathrm{SO}(3)$-connection. To illustrate the point, let us consider Bianchi type I models, $d s^{2}=-N^{2}\left(x^{0}\right)\left(d x^{0}\right)^{2}+g_{a b}\left(x^{0}\right) d x^{a} d x^{b}$, with the discrete symmetry $x^{3} \mapsto-x^{3}$. Then $g_{13}=g_{23}=0$. In terms of the triad $e_{(a) k}\left(x^{0}\right)$ and the connection $\omega_{(a) k}\left(x^{0}\right)$, the condition of invariance becomes

$$
\begin{array}{r}
e_{k}^{\prime(a)}=\chi^{(a)}{ }_{(b)} e_{k}^{(b)} \\
\omega_{k}^{\prime(a)}=\chi^{(a)}{ }_{(b)} \omega_{k}^{\prime(b)}+\frac{1}{2} \varepsilon^{(a b c)} \chi_{(b d)} \partial_{k} \chi^{-1}{ }^{(d)}
\end{array}
$$

where $e_{k}^{\prime(a)}=\left(e_{1}^{(a)}, e_{2}^{(a)},-e_{3}^{(a)}\right)$ and $\omega_{k}^{\prime(a)}=\left(\omega_{1}^{(a)}, \omega_{2}^{(a)},-\omega_{3}^{(a)}\right)$ 
Equation (34) shows that $\chi^{(a)}{ }_{(b)}$ has two eigenvalues equal to +1 and one equal to -1 . Hence it does not belong to $\mathrm{SO}(3)$ but rather to $\mathrm{O}(3)$. In this case, one must extend (34) in the following way:

$$
\begin{array}{r}
e_{k}^{\prime(a)}=\chi^{(a)}{ }_{(b)} e_{k}^{(b)} \\
\omega_{k}^{\prime(a)}=-\chi^{(a)}{ }_{(b)} \omega_{k}^{\prime(b)}+\frac{1}{2} \varepsilon^{(a b c)} \chi_{(b d)} \partial_{k} \chi^{-1(d)}{ }_{(c)}^{(d)}
\end{array}
$$

for elements $\chi^{(a)}{ }_{(b)}$ of $\mathrm{O}(3)$ which are not in the connected component to the identity. Indeed, $\omega_{k}^{\prime(a)}$ is a pseudo-vector for $\mathrm{O}(3)$ transformations.

In an appropriate basis, the matrix $\chi^{(a)}{ }_{(b)}$ reads

$$
\chi_{(b)}^{(a)}=\left(\begin{array}{ccc}
1 & 0 & 0 \\
0 & 1 & 0 \\
0 & 0 & -1
\end{array}\right)
$$

and is constant. The invariance condition implies then

$$
\begin{array}{r}
e_{3}^{(1)}=e_{3}^{(2)}=0, e_{1}^{(3)}=e_{2}^{(3)}=0 \\
\omega^{(1)} 1=\omega_{2}^{(1)}=0, \omega_{1}^{(2)}=\omega_{2}^{(2)}=0
\end{array}
$$

The variables remaining in the reduced variationnal principle for Bianchi type $\underline{\bar{I}}$ cosmologies with discrete symmetry $x^{3} \mapsto-x^{3}$ are thus:

(i) $e_{k}^{(a)}, \omega_{k}^{(3)} \quad(a=1,2 ; k=1,2)$,

(ii) $e_{3}^{(3)}, \omega_{3}^{(k)} \quad(a=1,2)$,

(iii) $N$ and $N^{k}$ (which drops out).

The gauge symmetries are (i) the redefinition of the time variable; and (ii) the $\mathrm{SO}(2)$-rotations in the (1)-(2) plane.

\section{Conclusion}

We have shown that there exists a natural definition of homogeneity preserving diffeomorphisms for Bianchi models such that none of the difficulties found in [3] arises. With that definition, the geometrical and dynamical concepts of gauge symmetries coincide irrespectively of the spatial topology. We 
have also verified the isomorphism of the Lagrangian covariant phase space with the Hamiltonian reduced phase space. Finally, we have discussed spatially homogeneous Ashtekar variables. We have illustrated how the general theory of invariant Yang-Mills connections apply. This theory is actually relevant in the discussion of all minisuperspace models formulated in terms of Ashtekar variables, and not just the Bianchi cosmological models with a three-dimensional simply transitive invariance group. In particular, one can generalize the considerations of [28] section $\bar{V}$ to the Ashtekar description.

\section{Acknowledgements}

We are grateful to Luca Bombelli for useful discussions. This work has been supported an part by the FNRS(Belgium) and the Commision of the European Communities.

\section{References}

[1] M.Henneaux, "Sur la géométrodynamique avec les champs spinoriels", chapter V, PhD Thesis (Brussels 1980).

[2] M.Henneaux,Ann. Inst. Henri Poincaré $\underline{34}(1981) 329 ; \quad$ see also M.Henneaux, Lett. Nuovo Cimento $\underline{38}(1983) 609$ (especially page 612).

[3] A.Ashtekar and J.Samuel, Class.Quant.Grav.ㅁ(1991)2191.

[4] R.T.Jantzen, Commun.Math.Phys. $\underline{64}(1979) 211$.

[5] M.A.H.Mac Callum, in "General Relativity: An Einstein Centenary Survey", S.W.Hawking and W.Israel eds, Cambridge University Press (Cambridge: 1979).

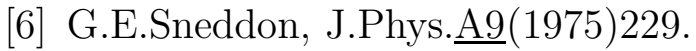

[7] A.H.Taub, Ann.Math. $\underline{53}$ (1951)472.

[8] M.Henneaux and C.Teitelboim, "Quantization of Gauge Systems", Princeton University Press(Princeton: 1992). 
[9] J.Kijowski and W.Szczyrba, Comm.Math.Phys.46(1976)183.

[10] E.Witten, Nucl.Phys.ㄹ276(1986)291.

[11] G.Zuckerman, Yale University Preprint(1986).

[12] A.Ashtekar, L.Bombelli and O.Reula, in "Analysis, Geometry and Mechanics: 200 years after Lagrange", eds.M.Francaviglia and D.Holm, Noth Holland, Amsterdam 1990.

[13] G.Barnich, M.Henneaux and C.Schomblond, Phys.Rev.D44(1991)R939.

[14] A.Ashtekar, Lectures on Non-Perturbative Canonical Gravity, World Scientific, Singapore (1991).

[15] C.Rovelli, Class.Quant.Grav.ㅁ(1991)1613.

[16] H.Kodama, Prog.Theor.Phys., 80(1988)1024.

[17] A.Ashtekar and J.Pullin, Ann.Israel.Phys.Soc. 9(1990)65

[18] P.Cordero and C.Teitelboim, Ann.Phys.(N.Y.) $\underline{100(1976) 603 ; ~}$ R.Benguria,P.Cordero and C.Teitelboim, Nucl.Phys.B122(1977)61.

[19] V.N.Romano,A.S.Schwarz and Yn.S.Tyupkin, Nucl.Phys. $\underline{\mathrm{B} 130}(1977) 209$.

[20] P.G.Bergmann and E.J.Flaherty, J.Math.Phys19(1978)212.

[21] A.Trautman, J.Phys.1ㅗ(1980)L1.

[22] A.S.Schwarz, Comm.Math.Phys. $\underline{56}(1977) 79$.

[23] M.Henneaux, J.Math.Phys.23(1982)830.

[24] J.Harnad,S.Shnider and L.Vinet, J.Math.Phys.20(1979)931.

[25] P.Forgács and N.S. Manton, Comm.Math.Phys. $\underline{56(1977) 79 .}$

[26] B.G.Schmidt, Comm.Math.Phys.15(1970)329.

[27] M.A.H.Mac Callum, in Cargèse lectures in Physics volume 6, ed.E.Schatzman, Gordan and Beach (New York 1973). 
[28] K.Kuchăr, Phys.Rev.D4(1971)955. 